The Cost of Improved Overview: An analysis of the Use of Electronic Whiteboards in Emergency Departments

Hertzum, Morten

Published in:

Proceedings of the INTERACT2017 Conference on Human-Computer Interaction

DOI:

10.1007/978-3-319-67684-5_25

Publication date:

2017

Document version

Peer reviewed version

Citation for published version (APA):

Hertzum, M. (2017). The Cost of Improved Overview: An analysis of the Use of Electronic Whiteboards in Emergency Departments. In Proceedings of the INTERACT2017 Conference on Human-Computer Interaction (Vol. LNCS 10514, pp. 402-410). Springer. https://doi.org/10.1007/978-3-319-67684-5_25 
Hertzum, M. (2017). The cost of improved overview: An analysis of the use of electronic whiteboards in emergency departments. In: Proceedings of the INTERACT2017 Conference on Human-Computer Interaction, Part II, LNCS 10514, pp. 402-410. Springer, Cham. The final version of the publication is available at Springer via http://dx.doi.org/doi:10.1007/978-3-31967684-5_25

\title{
The Cost of Improved Overview: An analysis of the Use of Electronic Whiteboards in Emergency Departments
}

\author{
Morten Hertzum \\ University of Copenhagen, Denmark \\ hertzum@hum.ku.dk
}

\begin{abstract}
Forming and maintaining an overview of an information space is key to competent action in many situations and often supported by overview displays. We investigate the cost of the improved overview associated with the introduction of electronic whiteboards in four emergency departments (EDs). In such a dynamic environment the work that goes into keeping the whiteboard current is, we contend, an indicator of the cost of maintaining an overview. On the basis of log data for the period 2012-2014 we find that the ED clinicians make an average of 1.91 whiteboard changes per minute to keep the whiteboard current. Performing these changes takes an estimated 6647 hours a year in each ED. While the whiteboard is well-like and has improved the clinicians' overview, our costof-overview estimation shows that it consumes substantial staff resources. This reflects the value the clinicians assign to having an overview but also reveals the amount of resources removed from other activities to maintain this overview.
\end{abstract}

Keywords: cost of use, overview formation, electronic whiteboard, healthcare

\section{Introduction}

Professionals in engineering, healthcare, management, and other dynamic domains are confronted with large amounts of information in their daily work and must be able to navigate it competently. Relatedly, common non-work activities involve monitoring continuously evolving social media for updates of interest and searching vast information spaces for items about specified topics. The sheer amount of information has made it challenging for users to maintain the overview necessary to navigate the information and has made overview displays an important part of many systems [1, 2]. One indication of the importance of overview displays is Shneiderman's [3] visual information-seeking mantra: "Overview first, zoom and filter, then details-ondemand”. Before users know where to zoom in and what to filter away, they first need an overview of the information space. However, an overview does not come for free. It is especially demanding to maintain an overview when the information space changes dynamically because the overview decays quickly and therefore has to be continuously updated to stay current. That is, while an initial overview may be necessary, it is not sufficient. This study investigates the amount of work involved in maintaining an overview of the dynamically evolving state of an emergency department (ED). 
EDs are the common entry point to hospitals for most patients with acute problems. In the ED the patients are assessed (triaged) to determine the severity of their problem [4]. Patients with high-severity problems are attended straight away to arrive at an initial diagnose and then transferred to the appropriate inpatient department for further treatment. Patients with low-severity problems may wait for hours before they are seen by a clinician. This patient flow means that most patients stay in the ED for a brief period only. But the patient volume is large, and every now and then it exceeds the available ED resources [5, 6]. Initial information about the patients' condition will tend to be rudimentary and additional information must be acquired quickly, reliably, and often under time pressure. Any oversight about a patient's condition may be detrimental to her health but this pressure toward detailed patient examinations must be balanced against the need to see a number of patients quickly. These work conditions create an environment in which it is important to have, and continuously maintain, an overview of the current state of the ED: Which patients are in more urgent need of attention? Which clinicians are responsible for which patients? What are the health problems of my current patients? What treatment should be administered to these patients? What is the status of this treatment? Are new patients about to arrive? And so forth.

We have for several years been involved in a project aiming to support ED clinicians in maintaining an overview of their work [e.g., 7, 8, 9]. This project has consisted of technically developing, organizationally implementing, and systematically evaluating an electronic whiteboard in the EDs in Region Zealand, one of the five healthcare regions in Denmark. The whiteboard (Fig. 1) supplements the electronic patient record by providing selected information about all patients currently in the ED. For each patient the whiteboard provides one row of information, including time of arrival, triage level, responsible physician, responsible nurse, working diagnose, current treatment activity, lab-test results, and - if relevant - the time at which the patient will be transferred to an inpatient department. The coordinating nurse plays a key role in keeping the whiteboard current but whiteboard updates are made by all physicians and nurses as well as by some other staff groups. In the present study we analyze almost three years of log data from the whiteboards in the four EDs of the region to assess the amount of work that goes into keeping the whiteboard current. We take this work as an indicator of the work-related cost of maintaining an overview.

It is important to note that the whiteboard has been successful in supporting the ED clinicians in forming and maintaining an overview of their work. In interviews
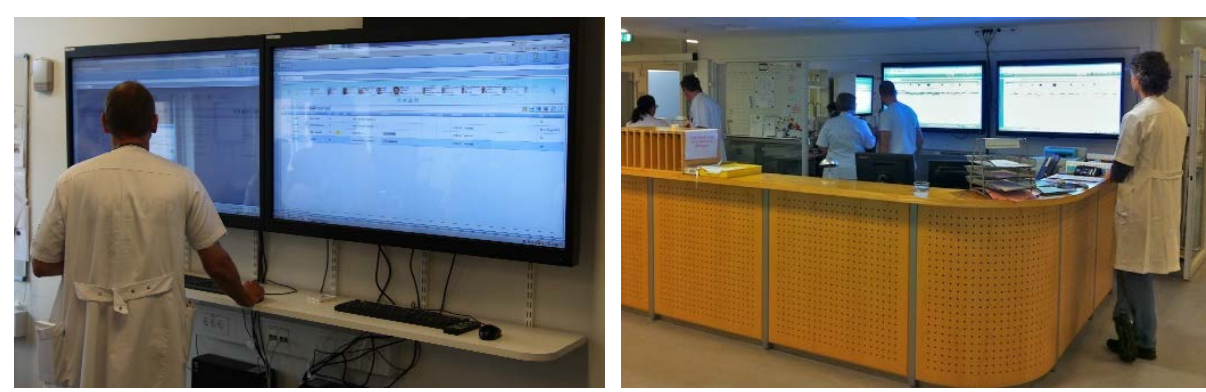

Fig. 1. The electronic whiteboard (a pair of 52-inch touch screens) in two of the EDs. 
conducted as part of our activities relating to the whiteboard a physician at ED3, for example, expressed that "It gives a great overview. I cannot imagine that we could do without it." More formally, a survey at ED1 and ED2 showed that the clinicians experienced an improvement in their overview of their work when the electronic whiteboard replaced the former dry-erase whiteboards [10]. In addition, before/after measurements at ED4 showed that the nurses' mental workload decreased at the beginning of their shifts when they form an overview of the ED [11]. That is, the work that goes into changing the whiteboard to keep it current has improved the clinicians' overview of their work and is, we contend, an indicator of the cost of the improved overview.

\section{The Notion of Overview}

Hornbæk and Hertzum [1] emphasize the distinction between overviews (displays in a user interface) and overviewing (a user's awareness of an aspect of an information space). While the whiteboard is an example of an overview display, it appears risky to assume that overviewing will ensue simply from attending to the whiteboard. Yet, Spence [12] and others take the term overview to imply that the user becomes aware of information in a rapid or even pre-attentive manner, that is without cognitive effort. This suggests that overviewing requires little more than periodically glancing at the whiteboard. Other researchers describe the process of acquiring an overview as more active, thereby suggesting that the user makes sense of the information space through active involvement with the overview display. For example, Bossen and Jensen [13, p. 257] tentatively define the process of achieving an overview as "how health care professionals arrive at a sufficiently informed, accountable and coherent understanding of a situation, so that they are capable of acting consciously and with confidence.” This definition states that to provide competent patient treatment the ED clinicians must form and maintain an overview based on which treatment decisions can be made. That is, overviewing is a central clinical activity. In addition, the definition suggests that users need to engage with the displayed information to make sense of it. Glancing at the whiteboard may be key to this engagement but it is not sufficient.

In order for a display to support its users in forming and maintaining an overview it must contain accurate information. While previous research on overviewing [1] - and related notions such as sensemaking [14] and situation awareness [15] - accentuates that in dynamic environments the users' understanding of the situation must evolve to stay current, it speaks less about the work that goes into keeping overview displays and other external representations current. In their investigation of the cost structure of sensemaking, Russell et al. [16] found that the main cost was associated with finding and encoding the relevant information. This activity amounted to over $75 \%$ of the total time even though it appears that the information space (a set of documents) was known and static. We contend that in a dynamic environment the continuous work of finding the relevant information and encoding it on an overview display is, by far, the biggest cost that comes with using the display to maintain an overview. 


\section{$3 \quad$ Method}

The four EDs in Region Zealand introduced the same electronic whiteboard in December 2009 (ED1), January 2010 (ED2), January 2011 (ED3), and May 2011 (ED4). The EDs were part of medium-sized hospitals that collectively served a population of approximately 820000 citizens. Some of the whiteboard data, such as labtest results, were automatically updated when new data became available, but most of the whiteboard data were entered and updated manually. This study is exclusively about the data that were entered and updated manually. Prior to conducting the study we obtained approval from the healthcare region.

\subsection{The Log Data}

All changes of the whiteboard content were automatically logged. For the purpose of this study the whiteboard vendor, Imatis, produced a version of the logs from which all patient names, clinician names, and other information that might identify persons had been removed. These anonymized log data covered the three-year period 2012-2014. However, we had to discard the periods January 2013 - January 2014 (ED1) and November 2013 - January 2014 (ED2-ED4) from the analysis because they contained long intervals of no data. After also removing 741 outliers (defined as ED visits longer than seven days, i.e. more than 50 times the median length of stay), the dataset comprised 380611 ED visits. The data for these visits consisted of more than 10 million log entries, each documenting a manually performed change of the whiteboard content. A log entry contained a timestamp, an event type, any values associated with the event, and a system-generated identifier of the visit to which the event pertained. For example, the event type 'TRIAGEChanged' along with the event value ' 5 ' showed that a clinician had changed the patient's triage level to 5 (indicating a life-threatening condition).

\subsection{Data Analysis}

We used the timestamp and the visit identifier of the log entries to determine the number of change events per minute and per patient. We also calculated the month-by-month evolution in the number of change events and their distribution onto day (08-16), evening (16-00), and night (00-08) shifts. These calculations served to assess whether the cost of the improved overview was evenly distributed across time.

To convert the number of change events into time spent we needed an estimate of the average duration of a change event. On the basis of an experiment with 18 clinicians from ED2, Rasmussen and Hertzum [17] found that it took the clinicians an average of 26.2 seconds to make a change on the whiteboard, including the time to log on to the whiteboard (by briefly holding a personal token onto a reader). Most whiteboard interactions consist of logons to make one or two changes. The clinicians in the experiment were experienced users of the whiteboard and they made fairly simple changes. To avoid overestimating the duration of a change event we set it to 20 seconds.

Before the introduction of the electronic whiteboard the EDs used dry-erase whiteboards. To provide an, admittedly rough, estimate of the added time involved in 
using the electronic rather than the dry-erase whiteboard we estimated that it took equally long to make a change on the electronic and dry-erase whiteboards and that the electronic whiteboard was changed $82 \%$ more often than the dry-erase whiteboard. Our basis for estimating that it took equally long to make changes on the two whiteboards was that, depending on the kind of change, Rasmussen and Hertzum [17] found either no difference in the time required to change the whiteboards or slightly longer times for the electronic whiteboard. Our basis for estimating that the electronic whiteboard was updated $82 \%$ more often than the dry-erase whiteboard was that Hertzum and Simonsen [7] arrived at this relationship between the number of changes on the two whiteboards in ED3. It should however be noted that the relationship was determined on the basis of few data, all of which from day shifts. That is, our estimate of the added time involved in using the electronic rather than the dry-erase whiteboard should be treated as merely suggestive.

\section{$4 \quad$ Results}

As a preamble to the analysis we note that all four EDs saw 30-40 thousand patients a year. That is, they were about equally busy.

\subsection{Number of Whiteboard Changes}

Table 1 shows the number of changes the ED clinicians made to the content of the whiteboard in the period covered by the dataset. The lower number of patients for ED1 was due to the shorter period for which we had data for this ED. While there was some variation across EDs, the general picture in all EDs was one of frequent changes. Across the four EDs the clinicians made an average of 26.63 whiteboard changes per patient, corresponding to an average of 1.91 changes per minute. Thus, the clinicians' improved overview of their work after the introduction of the electronic whiteboard was achieved by devoting a substantial amount of work to keeping the whiteboard current.

At the beginning of 2012 (the start of the dataset) the EDs had been using the whiteboard for between half a year and two years. Thus, it could be expected that the use of the whiteboard had had time to stabilize and that the clinicians had arrived at a work practice with a fairly standardized number of whiteboard changes per patient. Fig.

Table 1. Number of changes to the content of the whiteboard

\begin{tabular}{lcccc}
\hline ED & $\begin{array}{c}\text { Number of } \\
\text { change events }\end{array}$ & $\begin{array}{c}\text { ED visits } \\
\text { (i.e., patients) }\end{array}$ & $\begin{array}{c}\text { Changes } \\
\text { per patient }\end{array}$ & $\begin{array}{c}\text { Changes } \\
\text { per minute }\end{array}$ \\
\hline ED1 & 1905328 & 63759 & 29.88 & 1.92 \\
ED2 & 2669736 & 110479 & 24.17 & 1.85 \\
ED3 & 2200990 & 95308 & 23.09 & 1.53 \\
ED4 & 3360537 & 111065 & 30.26 & 2.34 \\
\hline Total & 10136591 & 380611 & 26.63 & 1.91 \\
\hline
\end{tabular}


2 disproves this expectation. All four EDs experienced an increasing trend in the work that went into obtaining the improved overview provided by the whiteboard. The trendlines (see Fig. 2) from a linear regression of the month-by-month evolution in changes per patient had a positive slope for all four EDs and explained 70\% (ED1), 69\% (ED2), 84\% (ED3), and 89\% (ED4) of the variance in the data. Because the increasing trend was in changes per patient it was not caused by a change in the number of patients. In addition, the trend occurred during a period with no increase in the number of clinicians. Rather, it appears that the clinicians gradually found more ways of using the whiteboard to improve their overview and that these new ways of working involved that the same number of clinicians made more frequent whiteboard changes.
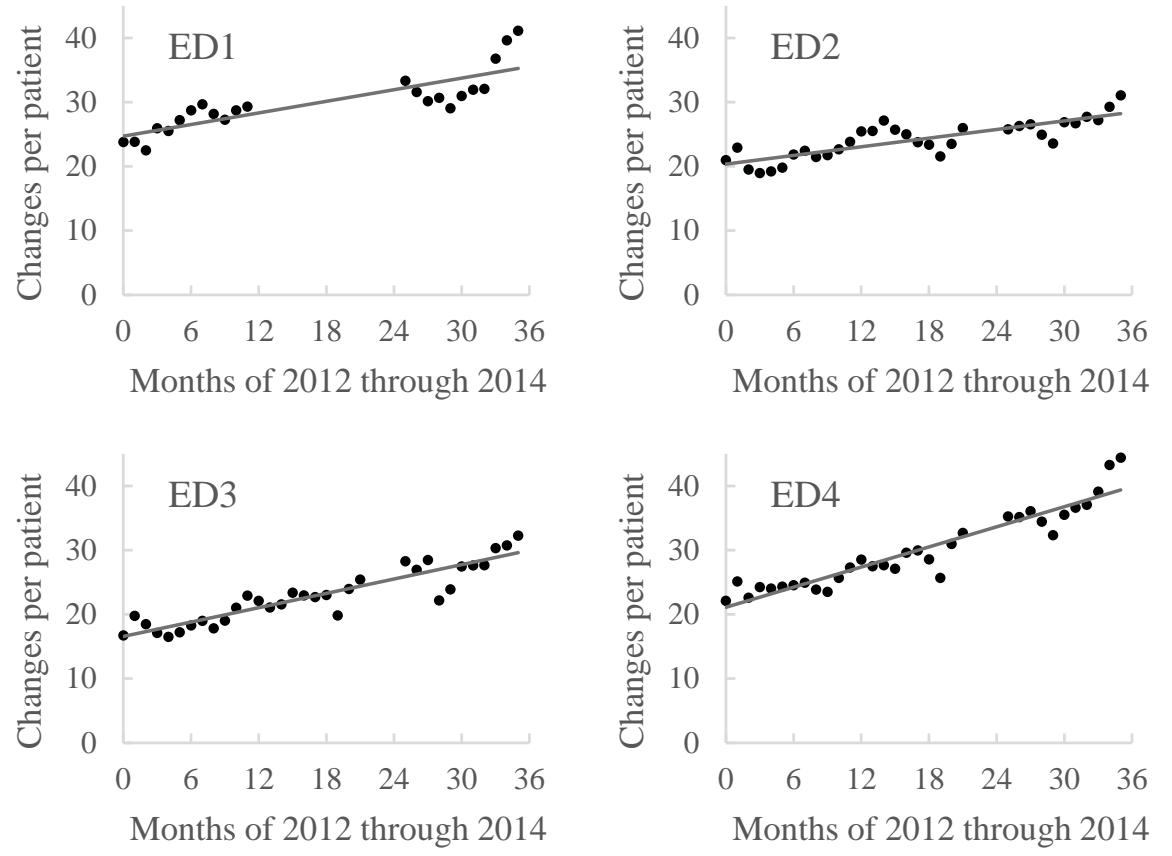

Fig. 2. Month-by-month evolution in the number of whiteboard changes per patient

Unsurprisingly, the changes of the whiteboard content were not evenly distributed across work shifts (Table 2). The clinicians changed the whiteboard content more frequently during day (08-16) and evening (16-00) shifts than during night (00-08) shifts because the number of patients was larger during day and evening shifts. Thus, most of the work that went into changing the whiteboard content was performed during the shifts in which the ED clinicians were most busy attending to patients. It is more noteworthy that the largest increase in the number of whiteboard changes (calculated by comparing the number of changes in December 2014 to that in January 2012) was also during the day and evening shifts, see Table 2. Presumably, the clinicians discovered more new ways of utilizing the whiteboard during the more busy day and 
evening shifts than during night shifts, thereby suggesting that the whiteboard offered the largest potential to improve the clinicians' overview of their work when the workload was high and it was challenging to maintain an overview. Table 2 also shows that the larger increase in whiteboard changes during day and evening shifts was mainly due to the two EDs that had introduced the whiteboard most recently (ED3 and ED4). Apparently, the whiteboard-related work practices in these two EDs had not yet stabilized in the beginning of 2012. Along with the trendlines in Fig. 2 this analysis suggests that the last part of the dataset is more representative of the number of whiteboard changes associated with obtaining an improved overview.

Table 2. Increase in whiteboard changes divided onto work shifts

\begin{tabular}{lccccccc}
\hline \multirow{2}{*}{ ED } & \multicolumn{3}{c}{ Changes per minute } & & \multicolumn{3}{c}{ Increase (Jan. 2012 to Dec. 2014) } \\
\cline { 2 - 3 } \cline { 7 - 8 } & Day & Evening & Night & & Day & Evening & Night \\
\hline ED1 & 2.84 & 2.10 & 0.82 & & $65 \%$ & $89 \%$ & $51 \%$ \\
ED2 & 2.71 & 2.11 & 0.74 & & $56 \%$ & $34 \%$ & $43 \%$ \\
ED3 & 2.24 & 1.78 & 0.57 & & $124 \%$ & $116 \%$ & $59 \%$ \\
ED4 & 3.31 & 2.71 & 0.98 & & $122 \%$ & $134 \%$ & $50 \%$ \\
\hline Average & 2.77 & 2.18 & 0.77 & & $92 \%$ & $93 \%$ & $51 \%$ \\
\hline
\end{tabular}

\subsection{Cost-of-Overview Estimation}

We used the last 12 months of data to estimate the annual cost of the clinicians' improved overview of their work, see Table 3. According to our estimates the EDs spent an average of 6647 hours updating the whiteboard during these 12 months, corresponding to an increase of 2991 hours compared to the time they previously spent updating the dry-erase whiteboards. The cost of the improved overview was similar at EDs 1, 2, and 3 and somewhat higher at ED4.

Table 3. Cost-of-overview estimation for 12 months of whiteboard use

\begin{tabular}{lccc}
\hline ED & $\begin{array}{c}\text { Number of } \\
\text { change events }\end{array}$ & $\begin{array}{c}\text { Hours spent } \\
\text { (estimated) }\end{array}$ & $\begin{array}{c}\text { Added hours } \\
\text { (estimated) }\end{array}$ \\
\hline ED1 & 1019331 & 5663 & 2548 \\
ED2 & 1084920 & 6027 & 2712 \\
ED3 & 1098681 & 6104 & 2747 \\
ED4 & 1582644 & 8792 & 3957 \\
\hline Average & & 6647 & 2991 \\
\hline
\end{tabular}

\section{$5 \quad$ Concluding Discussion}

The clinicians change the content of the whiteboard more than twice a minute during day and evening shifts and more than once every two minutes during night shifts. In the 
dynamic ED environment these changes are necessary to keep the whiteboard current. Importantly, the use of the whiteboard is merely recommended, not mandated, by hospital management; the ED clinicians spend time updating the whiteboard because they find that the improved overview justifies the time spent. We estimate that the time spent amounts to 6647 hours a year in each of the EDs or the equivalent of about 1.7 extra full-time clinicians compared to the previous use of dry-erase whiteboards.

The cost-of-overview estimate shows the large value the clinicians assign to having an overview of the state of the ED. It is also evident that this overview is a collaborative accomplishment: Multiple clinicians must contribute updates to keep the whiteboard current and, in return, the whiteboard supports multiple clinicians in forming and maintaining an overview. This collaborative aspect of overview has previously been investigated by, for example, Bossen and Jensen [13], who note that novice and experienced clinicians form an overview in quite different ways but still contribute to the collaborative accomplishment of an overview. Relatedly, several studies show that the value of whiteboards is, in part, that they create a place where people meet face to face and information is exchanged $[7,18,19]$. Meeting at the whiteboard is a valued occasion for consulting experienced colleagues, for obtaining details not on the whiteboard as well as for glancing at the whiteboard. In addition, the cost-of-overview estimate shows the potential of automating the whiteboard updates by deriving them from data in the electronic patient record. Such automation requires high-quality data because bypassing manual data entry also means bypassing a clinical judgement of whether the data make sense. However, automatic updates appear to be the only way to reduce the cost of overview while at the same time increasing the number of whiteboard changes in order to improve the overview further.

Two limitations should be remembered in interpreting the results of this study. First, the dataset is from one work domain and one country. While the four EDs provide evidence that the results are not peculiar to one hospital, we acknowledge that the results may be specific to healthcare and to Denmark. Second, the conversion of the number of change events to hours spent is no better than the estimate of the duration of the events and that of the ratio of electronic to dry-erase whiteboard changes. The latter estimate, in particular, is based on few empirical data. Much more research is needed to quantify the cost of overview. This study proposes that in dynamic environments the number of changes needed to keep an overview display current is indicative of this cost and shows that it may be substantial.

\section{Acknowledgements}

This study is part of the Clinical Communication project, which is a research and development collaboration between Roskilde University, University of Copenhagen, Imatis, and Region Zealand. The interview quote at the end of Section 1 is from an interview conducted in collaboration with Jesper Simonsen from Roskilde University. Special thanks are due to Rasmus Rasmussen at Imatis for making the anonymized version of the log data. 


\section{References}

1. Hornbæk, K., Hertzum, M.: The notion of overview in information visualization. International Journal of Human-Computer Studies 69(7\&8), 509-525 (2011).

2. Card, S.K., Mackinlay, J.D., Shneiderman, B. (eds.): Readings in information visualization: Using vision to think. Morgan Kaufmann, San Francisco, CA (1999).

3. Shneiderman, B.: The eyes have it: A task by data type taxonomy for information visualizations. In: Proceedings of the 1996 IEEE Conference on Visual Languages, pp. 336-343. IEEE Press, Los Alamitos, CA (1996).

4. Fitzgerald, G., Jelinek, G.A., Scott, D., Gerdtz, M.F.: Emergency department triage revisited. Emergency Medicine Journal 27(2), 86-92 (2010).

5. Moskop, J.C., Sklar, D.P., Geiderman, J.M., Schears, R.M., Bookman, K.J.: Emergency department crowding, part 1 - Concept, causes, and moral consequences. Annals of Emergency Medicine 53(5), 605-611 (2009).

6. Hoot, N.R., Aronsky, D.: Systematic review of emergency department crowding: Causes, effects, and solutions. Annals of Emergency Medicine 52(2), 126-136 (2008).

7. Hertzum, M., Simonsen, J.: Visual overview, oral detail: The use of an emergencydepartment whiteboard. International Journal of Human-Computer Studies 82, 2130 (2015).

8. Rasmussen, R., Fleron, B., Hertzum, M., Simonsen, J.: Balancing tradition and transcendence in the implementation of emergency-department electronic whiteboards. In: Molka-Danielsen, J., Nicolaisen, H.W., Persson, J.S. (eds.) Selected Papers of the Information Systems Research Seminar in Scandinavia 2010, pp. 73-87. Tapir Academic Press, Trondheim, NO (2010).

9. Rasmussen, R., Hertzum, M.: Visualizing the application of filters: A comparison of blocking, blurring, and colour-coding whiteboard information. International Journal of Human-Computer Studies 71(10), 946-957 (2013).

10. Hertzum, M.: Electronic emergency-department whiteboards: A study of clinicians' expectations and experiences. International Journal of Medical Informatics 80(9), 618-630 (2011).

11. Hertzum, M., Simonsen, J.: Effects of electronic emergency-department whiteboards on clinicians' time distribution and mental workload. Health Informatics Journal 22(1), 3-20 (2016).

12. Spence, R.: Information visualization: Design for interaction. Second edition. Prentice Hall, Harlow, England (2007).

13. Bossen, C., Jensen, L.G.: How physicians 'achieve overview': A case-based study in a hospital ward. In: Proceedings of the CSCW2014 Conference on Computer Supported Cooperative Work \& Social Computing, pp. 257-268. ACM Press, New York (2014).

14. Weick, K.E., Sutcliffe, K.M., Obstfeld, D.: Organizing and the processs of sensemaking. Organization Science 16(4), 409-421 (2005).

15. Endsley, M.R.: Toward a theory of situation awareness in dynamic systems. Human Factors 37(1), 32-64 (1995). 
16. Russell, D.M., Stefik, M.J., Pirolli, P., Card, S.K.: The cost structure of sensemaking. In: Proceedings of the INTERCHI1993 Conference on Human Factors in Computing Systems, pp. 269-276. ACM Press, New York (1993).

17. Rasmussen, R., Hertzum, M.: Consider the details: A study of the reading distance and revision time of electronic over dry-erase whiteboards. In: DHRS2012: Proceedings of the Danish HCI Research Symposium, pp. 24-27. University of Southern Denmark, Sønderborg, DK (2012).

18. Scupelli, P., Xiao, Y., Fussell, S.R., Kiesler, S., Gross, M.D.: Supporting coordination in surgical suites: Physical aspects of common information spaces. In: Proceedings of the CHI 2010 Conference on Human Factors in Computing Systems, pp. 1777-1786. ACM Press, New York (2010).

19. Whittaker, S., Schwarz, H.: Meetings of the board: The impact of scheduling medium on long term group coordination in software development. Computer Supported Cooperative Work 8(3), 175-205 (1999). 